\title{
Transient Simulation of On-Chip Transmission Lines via Exact Pole Extraction
}

\author{
Guoqing Chen and Eby G. Friedman \\ Department of Electrical and Computer Engineering \\ University of Rochester \\ Rochester, New York 14627 \\ guchen,friedman@ece.rochester.edu
}

\begin{abstract}
An accurate and efficient solution for the transient response at the far end of a transmission line is proposed in this paper. Unlike approximating the poles by truncating the transfer function or matching moments, the exact poles of an interconnect system are analytically extracted. Excellent match is observed between the proposed method and Spectre simulations. With two pairs of poles, the average error for the $50 \%$ delay is $1 \%$. Higher accuracy can be obtained with additional pairs of poles. The computational complexity of the model is proportional to the number of pole pairs.
\end{abstract}

\section{INTRODUCTION}

With the scaling of CMOS technology, interconnect has become a dominant factor in high performance integrated circuits (ICs). Due to higher signal frequencies and longer wire lengths, on-chip interconnects exhibit significant transmission line behavior. An efficient solution to analyze transmission lines is therefore critical to the IC design process.

Sakurai presented in [1] an accurate closed-form solution for distributed $R C$ interconnect based on a single pole approximation. By truncating the transfer function, multi-pole models have been proposed in the last decade to capture the effect of inductance [2], [3]. In [4], the solution for an open-ended interconnect with a step input signal is rigorously developed. This solution, however, is computationally complicated and not suitable for an exploratory design process. In [5], a traveling wave analysis (TWA) model has been presented, where the key points of the waveform are determined with a three-pole model and linear or $R C$ approximations are used to connect these key points to construct the waveform. Similar to this approach, a piecewise linear (PWL) model is presented in [6], where the key points and slopes are more accurately determined with the model described in [4], and straight lines are used to construct the signal waveforms in different time regions. In both of these papers, the output response is divided into a number of time regions where the waveform expressions for each of the regions are different, making the models less compact.

This research is supported in part by the Semiconductor Research Corporation under Contract 2004-TJ-1207, the National Science Foundation under Contract Nos. CCR-0304574 and CCF-0541206, grants from the New York State Office of Science, Technology \& Academic Research to the Center for Advanced Technology in Electronic Imaging Systems, and by grants from Intel Corporation, Eastman Kodak Company, Intrinsix Corporation, and Freescale Semiconductor Corporation.

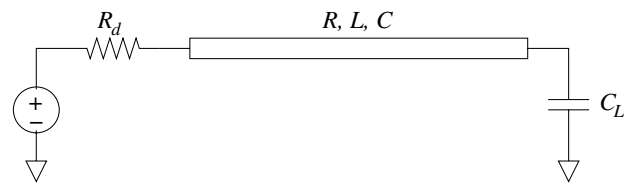

Fig. 1. Distributed interconnect with a lumped capacitive load and driver resistance.

In this paper, a new method for computing the far end response of a transmission line is proposed. The proposed model is based on a direct pole extraction of the exact transfer function of a transmission line, rather than approximating the poles by truncating the transfer function [2], [3] or matching the moments [7]. Flexible tradeoffs between accuracy and efficiency can be achieved. The rest of the paper is organized as follows. In Section II, the poles of an $R L C$ interconnect system without driver resistance are determined in closed form. In Section III, an interconnect system with general circuit parameters is solved. Finally, some conclusions are offered in Section IV.

\section{Distributed $R L C$ INTERCONNECT Without DRIVER RESISTANCE}

For a distributed $R L C$ interconnect driven by a voltage source with a driver resistance $R_{d}$ and loaded with a lumped capacitance $C_{L}$, as shown in Fig. 1, the transfer function is [8]

$H(s)=\frac{1}{\left(1+R_{d} C_{L} s\right) \cosh (\theta)+\left(R_{d} / Z_{c}+Z_{c} C_{L} s\right) \sinh (\theta)}$,

where $\theta=\sqrt{(R+L s) C s}$ and $Z_{c}=\sqrt{(R+L s) / C s}=$ $\theta / C s . R, L$, and $C$ are the resistance, inductance, and capacitance of the interconnect.

The poles of (1) can be solved if $R_{d}=0$. When $R_{d}=0$, (1) simplifies to

$$
H(s)=\frac{1}{\cosh (\theta)+C_{T} \theta \sinh (\theta)} .
$$

Let $F(s)=1 / H(s)$. Observe that $\theta$ needs to be an imaginary number to make $F(s)$ zero. Assume $\theta=j x$, where $x$ is a real number. The poles of the transfer function should satisfy

$$
\cos x-C_{T} x \sin x=0 .
$$


By approximating $\sin x$ and $\cos x$ with a Taylor series expansion around $n \pi, n=0,1,2, \cdots, x$ can be solved as

$$
x_{n}= \begin{cases}\sqrt{\frac{\frac{1}{2}+C_{T}-\sqrt{C_{T}^{2}+\frac{1}{3} C_{T}+\frac{1}{12}}}{\frac{1}{3} C_{T}+\frac{1}{12}}}, & n=0 \\ \frac{\left(1+C_{T}\right) n \pi+\sqrt{\left(C_{T} n \pi\right)^{2}+2+4 C_{T}}}{1+2 C_{T}}, & n \geq 1 .\end{cases}
$$

Note that when $C_{T}$ approaches zero, (3) becomes $\cos x=0$, and the solution $x_{n}$ approaches $(n+1 / 2) \pi$. In order to capture this trend, (4) is revised as

$$
x_{n}= \begin{cases}\sqrt{\frac{\frac{1}{2}+C_{T}-\sqrt{C_{T}^{2}+\frac{1}{3} C_{T}+\frac{1}{11.54}}}{\frac{1}{3} C_{T}+\frac{1}{12}}}, & n=0 \\ \frac{\left(1+C_{T}\right) n \pi+\sqrt{\left(C_{T} n \pi\right)^{2}+\frac{\pi^{2}}{4}+4 C_{T}}}{1+2 C_{T}}, & n \geq 1 .\end{cases}
$$

The poles of the transfer function can be obtained from the following expression,

$$
L C s^{2}+R C s=\theta^{2}=-x_{n}^{2}, n=0,1,2, \cdots .
$$

Each $x_{n}$ corresponds to a pair of poles,

$$
p_{n, \pm}=\frac{-R C \pm \sqrt{R^{2} C^{2}-4 L C x_{n}^{2}}}{2 L C} .
$$

The residue of the corresponding poles $k_{n, \pm}$ can be solved as

$$
\begin{aligned}
k_{n, \pm} & =\lim _{s \rightarrow p_{n, \pm}} \frac{s-p_{n, \pm}}{F(s)}=\frac{1}{F^{\prime}\left(p_{n, \pm}\right)} \\
& =\frac{ \pm 2 x_{n}}{D\left[\left(1+C_{T}\right) \sin x_{n}+C_{T} x_{n} \cos x_{n}\right]}
\end{aligned}
$$

where $D=\sqrt{R^{2} C^{2}-4 L C x_{n}^{2}}$.

From the poles and corresponding residues, the transfer function can be represented as

$$
H(s)=\sum_{i} \frac{k_{i}}{s-p_{i}},
$$

where $i$ is the index covering all of the poles. Consider a wire structure example as shown in Fig. 2. The interconnect parameters per unit length are $R_{\text {int }}=12.24 \mathrm{~m} \Omega / \mu \mathrm{m}, L_{i n t}=$ $0.74 \mathrm{pH} / \mu \mathrm{m}$, and $C_{\text {int }}=0.266 \mathrm{fF} / \mu \mathrm{m}$. The amplitude of the transfer function obtained from (9) is compared with the exact transfer function in Fig. 3. In this figure, $m$ is the number of pole pairs. As shown in the figure, the analytic transfer function converges to the exact transfer function with increasing $m$. From (9), the step response and ramp response at the far end of an interconnect can be determined with an inverse Laplace transform.

\section{Distributed $R L C$ INTERCONNECT With DRIVER RESISTANCE}

In [6], the circuit model as shown in Fig. 1 is mapped into an open-ended interconnect system by matching the moments. Similarly, the interconnect system with a non-zero driver resistance can also be mapped into a system without a driver

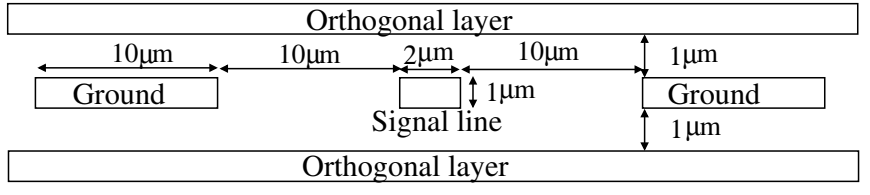

Fig. 2. Wire geometry of an example circuit, where the signal wire is shielded by two ground lines.

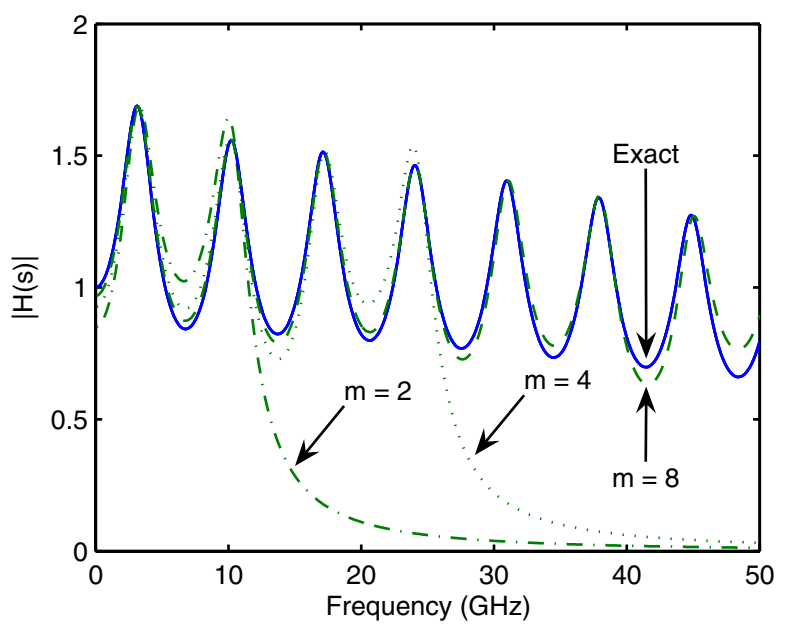

Fig. 3. Comparison between the analytic expression (9) and the exact transfer function. The wire length is $5 \mathrm{~mm}$ and the load capacitance is $C_{L}=50 \mathrm{fF}$.

resistance. Consider a step signal at the input of the circuit shown in Fig. 1. The height of the initial step at the driver output is $V_{d d} Z_{0} /\left(R_{d}+Z_{0}\right)$, where $Z_{0}=\sqrt{L / C}$ is the characteristic impedance of a lossless line. As described in [9], the attenuation coefficient of a transmission line saturates with increasing frequency to the asymptotic value $R /\left(2 Z_{0}\right)$. Assume the interconnect resistance of the new system (without a driver resistance) is $R^{\prime}$ and the load capacitance is $C_{L}^{\prime}$. By matching the amplitude of the initial propagating wave,

$$
V_{d d} \frac{Z 0}{R_{d}+Z_{0}} e^{-\frac{R}{2 Z_{0}}}=V_{d d} e^{-\frac{R^{\prime}}{2 Z_{0}}},
$$

$R^{\prime}$ can be obtained as

$$
R^{\prime}=R+2 Z_{0} \log \left(1+\frac{R_{d}}{Z_{0}}\right)
$$

By matching the first moments of the two systems, $C_{L}^{\prime}$ can be obtained as

$$
C_{L}^{\prime}=\frac{R_{d}\left(C_{L}+C\right)+R\left(0.5 C+C_{L}\right)}{R^{\prime}}-0.5 C .
$$

After this conversion, the pole expressions described in Section II can be applied. The location of the low order poles obtained analytically is compared with the location of the exact poles in Fig. 4. From the figure, note that there is a one-to-one mapping between the approximated poles and the exact poles. The real pole without an arrow in Fig. 4 should be mapped to a real pole which is out of the range of the figure. From these approximated poles, the exact poles can be obtained through 


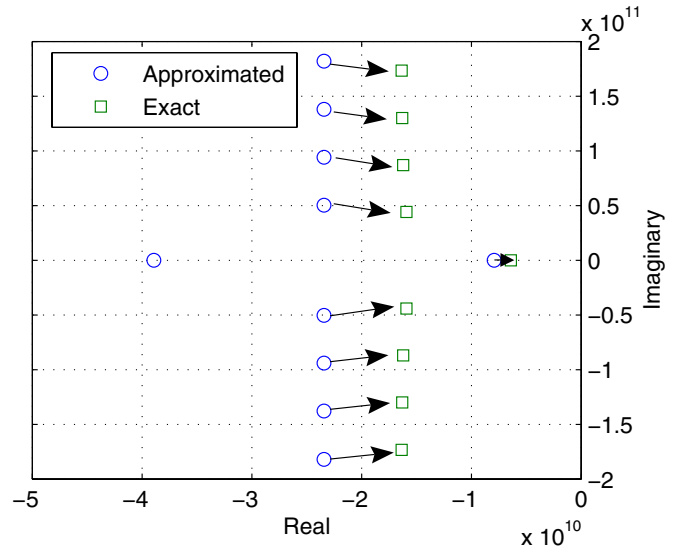

Fig. 4. Mapping between the approximated poles and the exact poles, $R_{d}=$ $100 \Omega$.

the Newton-Raphson method, permitting the accuracy of the model to be significantly improved. In general, the number of iterations required for convergence is less than five.

Special attention needs to be paid to those real poles when applying the Newton-Raphson method. For example, the Newton-Raphson process starting from the approximated pole $-3.892 \times 10^{10}$ (the left real pole as shown in Fig. 4) incorrectly converges to the exact pole $-6.396 \times 10^{9}$ rather than converges to the exact pole outside the range of the figure. In order to distinguish this case from the double real pole case, the following condition needs to be evaluated. If $p$ is a double real pole of the system, $p$ satisfies the following expression,

$$
\lim _{s \rightarrow p} \frac{F(s)}{s-p}=F^{\prime}(p)=0 .
$$

For systems with multiple real poles, the system is dominated by the real pole with the smallest magnitude. The effect of the other real poles can be ignored, unless these poles are close to the dominant pole. The distance between the other real poles and the dominant real pole is related to the value of $F^{\prime}\left(p_{d}\right)$, where $p_{d}$ is the dominant pole. If there is another pole $p_{x}$ which is close to $p_{d}, F^{\prime}\left(p_{d}\right)$ should be small. When $p_{x}$ approaches $p_{d}$, the value of $F^{\prime}\left(p_{d}\right)$ approaches zero. In the limit, $p_{x}=p_{d}, p_{d}$ is a double pole, and $F^{\prime}\left(p_{d}\right)=0$, as expressed in (13).

Pseudo-code for generating the exact poles of a single interconnect system is shown in Fig. 5. In Fig. 5, the variable over_damped is used to indicate whether the system is overdamped or not. For overdamped systems, the higher order real poles (with $n>0$ ) are ignored. A threshold value $F_{t h}$ is set to $F^{\prime}(p)$, which is used to indicate the distance between other high order real poles and the dominant real pole. After the dominant real pole (if the system has real poles, the dominant real pole is always $\left.p_{0,+}\right)$ is determined, $F^{\prime}\left(p_{0,+}\right)$ is evaluated. $F(s)$ can be represented by the poles as

$$
F(s)=\prod_{n=0}^{\infty}\left(1-\frac{s}{p_{n,+}}\right)\left(1-\frac{s}{p_{n,-}}\right) .
$$

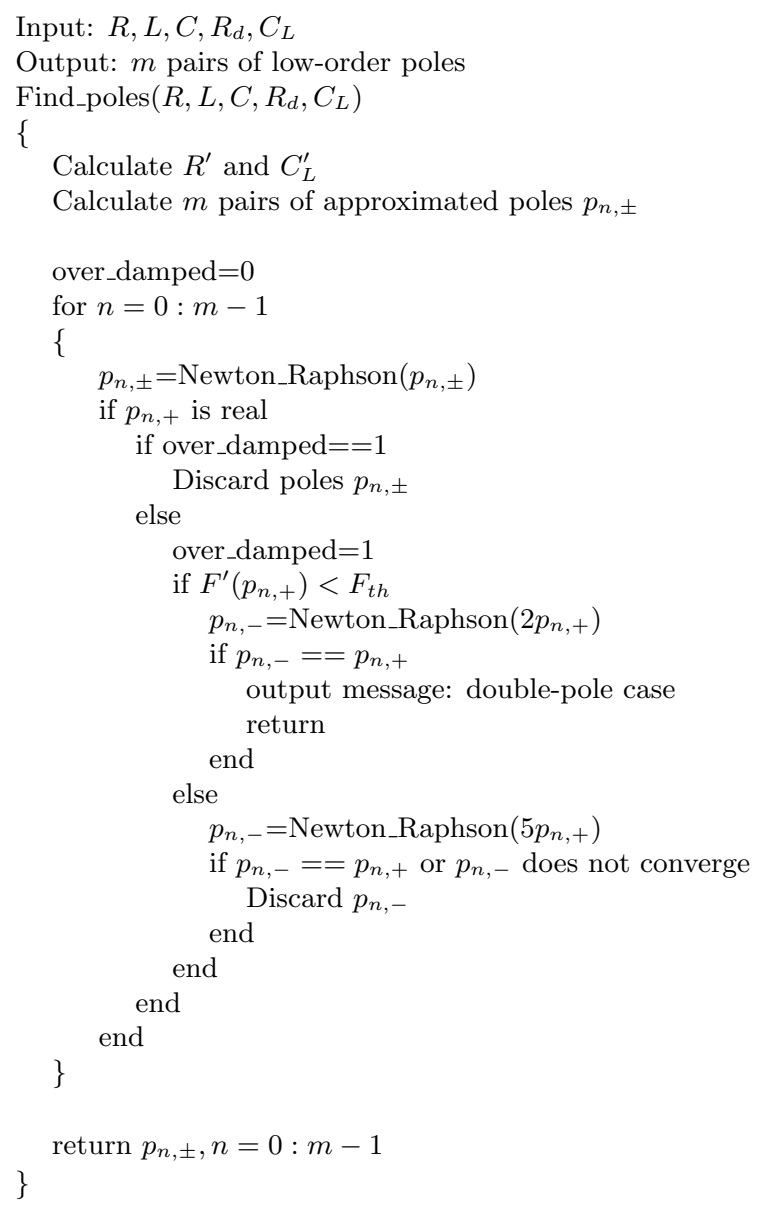

Fig. 5. Pseudo-code for computing the exact poles. The function Newton_Raphson ( ) is the Newton-Raphson converging process starting with the input argument.

From (14),

$$
\begin{aligned}
F^{\prime}\left(p_{0,+}\right) & =\frac{-1}{p_{0,+}}\left(1-\frac{p_{0,+}}{p_{0,-}}\right) \prod_{n=1}^{\infty}\left(1-\frac{p_{0,+}}{p_{n,+}}\right)\left(1-\frac{p_{0,+}}{p_{n,-}}\right) \\
& <\frac{-1}{p_{0,+}}\left(1-\frac{p_{0,+}}{p_{0,-}}\right) .
\end{aligned}
$$

If $\left|p_{0,-}\right|>2\left|p_{0,+}\right|, F^{\prime}\left(p_{0,+}\right)<-0.5 / p_{0,+}$. With some overhead, $F_{t h}$ is determined as $-0.3 / p_{0,+}$. If $F^{\prime}\left(p_{0,+}\right)<F_{t h}$, which means pole $p_{0,-}$ is close to $p_{0,+}$, a Newton_Raphson process is launched from point $2 p_{0,+}$ to determine $p_{0,-}$. Otherwise, the Newton_Raphson process is launched from point $5 p_{0,+}$ to determine $p_{0,-}$. If the process does not converge or incorrectly converges to $p_{0,+}$, meaning the true value of $\left|p_{0,-}\right|$ is greater than $5\left|p_{0,+}\right|$, the effect of $p_{0,-}$ can be ignored. For the double pole case, the process of solving the residue requires the second order derivative of $F(s)$, which is complicated. The pseudo-code shown in Fig. 5 produces an output message if a double pole occurs. In this case, a small change in the circuit parameters can avoid a double pole, while the effect on the output signal waveform caused by this parameter change cannot be distinguished. 


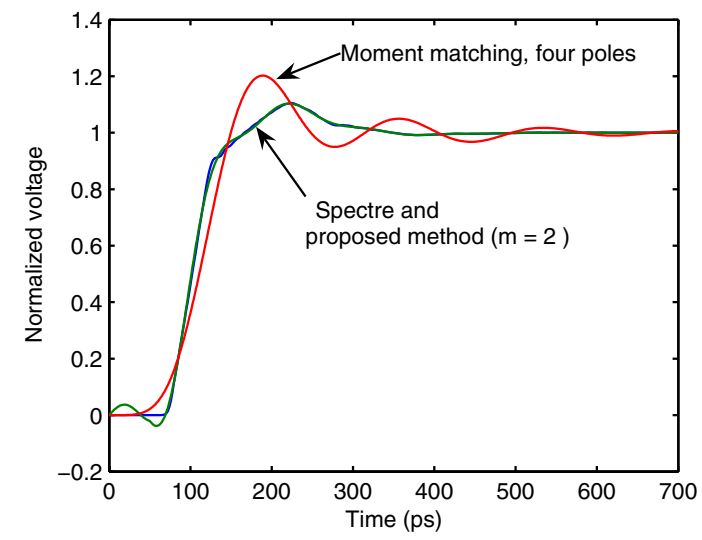

Fig. 6. Transient response of a transmission line obtained with the proposed analytic method, moment matching method, and Spectre simulations. $t_{r}=$ $50 \mathrm{ps}, C_{L}=50 \mathrm{fF}, l=5 \mathrm{~mm}$, and $R_{d}=20 \Omega$.

After the exact poles are extracted, a step or ramp response is constructed. The waveform obtained from the proposed method is compared with Spectre simulations and the moment matching method with four poles in Fig. 6. In the Spectre simulation, the transmission line is modeled as a series of $\pi$-shaped $R L C$ segments. Each segment is $10 \mu \mathrm{m}$ long. As shown in Fig. 6, the proposed method provides a much higher accuracy than the moment matching method with the same number of poles. To achieve an accuracy similar to the proposed method $(m=2)$, twelve poles are required in the moment matching method, resulting in a much higher computational complexity.

The $50 \%$ delay and $10 \%$-to-90\% output rise time obtained from the proposed model are compared in Fig. 7 with Spectre simulations for different input rise times (the input rise time is determined from 0 to $V_{d d}$ ). Since the signal delay is generally determined by the low frequency components, two pairs of poles provide a sufficiently accurate delay estimation. The average error is $1 \%$ for different input rise times. For the output rise time, the error is greater for smaller input rise times. The error decreases with increasing input rise time, since the output rise time is closely related to the high frequency components (a signal with a shorter rise time consists of additional high frequency components). The average error with two pairs of poles is $9.5 \%$ for the output rise time. When the number of pole pairs increases to ten, the average error decreases to $2 \%$. The computational complexity of the proposed method is approximately proportional to the number of pole pairs. These experiments have been performed on a SunBlade1500 workstation. The time required for Spectre to perform a $700 \mathrm{ps}$ transient simulation (250 time steps) is $1.8 \mathrm{~s}$. The proposed model is implemented with Matlab. The run time is $3.1 \mathrm{~ms}$ for $m=2$ and $10.9 \mathrm{~ms}$ for $m=10$.

\section{Conclusions}

By extracting the exact poles, an efficient method has been proposed in this paper for determining the transient output

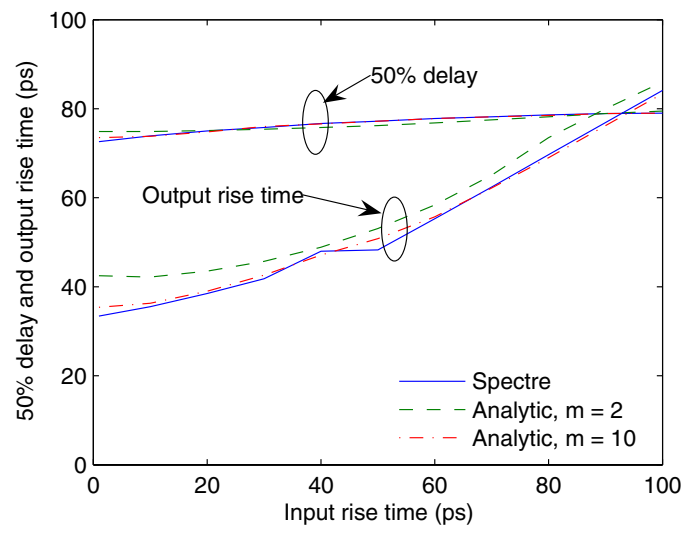

Fig. 7. Comparison of the $50 \%$ delay and $10 \%$-to- $90 \%$ output rise time obtained from the proposed model and Spectre simulations. $R_{d}=20 \Omega$, $C_{L}=50 \mathrm{fF}$, and $l=5 \mathrm{~mm}$.

response of a distributed $R L C$ interconnect. As demonstrated in the paper, two pairs of poles can provide an accurate delay estimate exhibiting an average error of $1 \%$ as compared with Spectre simulations. For high frequency related waveform properties, such as the rise time, an average error of $2 \%$ can be obtained with ten pairs of poles. The computational complexity of the proposed method is proportional to the number of pole pairs.

\section{REFERENCES}

[1] T. Sakurai, "Closed-Form Expressions for Interconnection Delay, Coupling, and Crosstalk in VLSI's," IEEE Transactions on Electron Devices, Vol. 40, No. 1, pp. 118-124, January 1993.

[2] A. B. Kahng and S. Muddu, "An Analytical Delay Model for $R L C$ Interconnects," IEEE Transactions on Computer-Aided Design of Integrated Circuits and Systems, Vol. 16, No. 12, pp. 1507-1514, December 1997.

[3] K. Banerjee and A. Mehrotra, "Accurate Analysis of On-Chip Inductance Effects and Implications for Optimal Repeater Insertion and Technology Scaling," Proceedings of the IEEE Symposium on VLSI Circuits, pp. 195198, June 2001.

[4] J. A. Davis and J. D. Meindl, "Compact Distributed $R L C$ Interconnect Models-Part I: Single Line Transient, Time Delay, and Overshoot Expressions," IEEE Transactions on Electron Devices, Vol. 47, No. 11, pp. 2068-2077, November 2000.

[5] Y. Eo, J. Shim, and W. R. Eisenstadt, "A Traveling-Wave-Based Waveform Approximation Technique for the Timing Verification of Single Transmission Lines," IEEE Transactions on Computer-Aided Design of Integrated Circuits and Systems, Vol. 21, No. 6, pp. 723-730, June 2002.

[6] J. Chen and L. He, "Piecewise Linear Model for Transmission Line With Capacitive Loading and Ramp Input," IEEE Transactions on ComputerAided Design of Integrated Circuits and Systems, Vol. 24, No. 6, pp. 928-937, June 2005.

[7] L. T. Pillage and R. A. Rohrer, "Asymptotic Waveform Evaluation for Timing Analysis," IEEE Transactions on Computer-Aided Design of Integrated Circuits and Systems, Vol. 9, No. 4, pp. 352-366, April 1990.

[8] G. Chen and E. G. Friedman, "An $R L C$ Interconnect Model Based on Fourier Analysis," IEEE Transactions on Computer-Aided Design of Integrated Circuits and Systems, Vol. 24, No. 2, pp. 170-183, February 2005.

[9] Y. I. Ismail, E. G. Friedman, and J. L. Neves, "Figures of Merit to Characterize the Importance of On-Chip Inductance," IEEE Transactions on Very Large Scale Integration (VLSI) Systems, Vol. 7, No. 4, pp. 442449, December 1999. 